\title{
Sixty Years of \\ Double Beta Decay
}

From Nuclear Physics to Beyond Standard Model Particle Physics 
This page is intentionally left blank 


\section{Sixty Years of \\ Double Beta Decay \\ From Nuclear Physics to Beyond \\ Standard Model Particle Physics}

H. V. Klapdor-Kleingrothaus

Max-Planck-Institut für Kernphysik, Germany 


\section{Published by}

World Scientific Publishing Co. Pte. Ltd.

P O Box 128, Farrer Road, Singapore 912805

USA office: Suite 1B, 1060 Main Street, River Edge, NJ 07661

UK office: 57 Shelton Street, Covent Garden, London WC2H 9HE

\section{British Library Cataloguing-in-Publication Data}

A catalogue record for this book is available from the British Library.

\section{SIXTY YEARS OF DOUBLE BETA DECAY: FROM NUCLEAR PHYSICS TO BEYOND STANDARD MODEL PARTICLE PHYSICS}

Copyright $\odot 2001$ by World Scientific Publishing Co. Pte. Ltd.

All rights reserved. This book, or parts thereof, may not be reproduced in any form or by any means, electronic or mechanical, including photocopying, recording or any information storage and retrieval system now known or to be invented, without written permission from the Publisher.

For photocopying of material in this volume, please pay a copying fee through the Copyright Clearance Center, Inc., 222 Rosewood Drive, Danvers, MA 01923, USA. In this case permission to photocopy is not required from the publisher.

ISBN 981-02-3779-0

Printed in Singapore by Uto-Print 


\section{Preface}

This book describes the history of double beta decay and its potential as research tool in particle physics. Double beta decay was investigated theoretically for the first time shortly after the introduction of the neutrino. Its history is related with fundamental problems and discoveries of particle physics such as lepton-number conservation, parity non-conservation, and gauge theories. Being considered in the beginning as a purely nuclear physics problem, it was understood early as the most sensitive means of probing the Majorana mass of the neutrino. Only rather late, in the early eighties, far after the experimental discovery of the neutrino, and after the introduction of gauge theories, its potential to determine quantitatively the (Majorana) mass of the neutrino was understood. The sensitivity of double beta decay experiments has reached now a level, at which it starts to 'dramatically constrain the form of the neutrino mass matrix' [Geo2000]. 'The at present most sensitive experiment already now excludes, as various theoretical papers since 1997 show, the small angle $M S W$ solution of the solar neutrino problem, if neutrinos are degenerate, and also according to some papers, with some reservation, the large angle $M S W$ solution, in this case, 'forcing us into the vacuum oscillation solution' [Ell99a].

A nice confirmation could be that recent Superkamiokanda solar neutrino results also claim, that the small angle MSW solution, (as well as the vacuum oscillation solution, and oscillation into sterile neutrinos) are ruled out with 95\% c.l. [Suz2000] (although some authors also make less definite conclusions [Gon2000]). Favoured remain the large angle solution and the $L O W$ solution. On the other hand it becomes visible that the various solar and other neutrino oscillation experiments may not finally be able to alone solve the problem of the neutrino mass matrix which makes double beta decay indispensable in helping to solve this problem (and in particular the problem of fixing an absolute mass scale).

For this purpose future double beta experiments with strongly increased sensitivity are required such as GENIUS (and other experiments prompted by this proposal). The proposal of GENIUS in 1997 has given a great impact to the field of double beta decay, particularly also to deeper theoretical investigations of the potential of the field for neutrino physics and other beyond standard model physics. 
Future experiments at the sensitivity level of GENIUS would help to discriminate various neutrino mass scenarios, among them the large mixing angle MSW solution. By using it as a real time solar neutrino detector for pp neutrinos, GENIUS also could probe the $L O W$ solution. As a by-product it will give the chance to search for cold dark matter with unprecedented sensitivity.

In recent years double beta decay has become ONE OF THE MOST IMPORTANT FIELDS OF NON-ACCELERATOR PARTICLE PHYSICS, by contributing to probing also other physics beyond the standard model in various directions already now on the TeV scale, where new physics should manifest itself.

Future experiments such as GENIUS will have the potential, to probe the multi$\mathrm{TeV}$ range for other beyond standard model topics such as supersymmetry, compositeness, leptoquarks, violation of Lorentz invariance, equivalence principle, etc. They could yield important complementary information to the physics to be expected from future high-energy colliders and satellite experiments.

The book does not intend to give all historical details. If in some cases the contributions of some researchers to the progress of the field may not have been always properly weighted, this may be not only the fault of the editor, first because of space restrictions, but also - and more important - since some publishers did not allow reprinting some important articles from their journals. The editor regrets this deeply. Another natural problem of such a type of book is, that the usually most valuable review articles can be reprinted only to a limited extent because of space reasons. We gave in these cases, as also for books and for some other important articles which could not be fully included, as a hint and help for the reader at least the first pages. In the Introductory Chapter I, which gives an overview of the history, status and perspectives of the field, for the convenience of the reader, articles which are fully reprinted in this book, are marked with an asterisk $\left({ }^{*}\right)$, followed by a Roman number indicating the subchapter of Chapter 2 , where it can be found. In case that only the first page(s) are reprinted, the article is marked with two asterisks $(* *)$.

It is hoped, that in any case the lines of development of the field are clearly shown, and also, in particular, the great future potential of this kind of nonaccelerator particle physics becomes transparent. This is the main aim of this book.

We hope that the book will be useful and stimulating for students and researchers in the field of particle physics.

It is the pleasure of the editor to thank his colleagues from the Heidelberg Double Beta and Dark Matter Group, and the many scientific colleagues and guests from many countries, for many years of fruitful and pleasant collaboration on the subject of this book.

The editor is most indebted to Dr. Irina Krivosheina, for giving the inspiration for the edition of this book and for her invaluable help in its realization.

H.V. Klapdor-Kleingrothaus Heidelberg, 5 July, 2000 


\section{Acknowledgments}

The author and publisher gratefully acknowledge permission to reproduce previously published material, as granted by authors and publishers, and as indicated by citations. They have attempted to trace the copyright holders of all material reproduced from all sources and apologize to any copyright holders whose prior permission might not have been obtained.

We are grateful in particular to American Association for the Advancement of Science: Science Associate Publishers, American Physical Society: Phys. Rev, Phys. Rev. Lett. Elsevier Science Publishers B.V.: Phys. Lett., Nucl. Phys., for the permission to reprint the authors own articles

European Physical Society: Europhysics Letters, Europhysics News

Gordon and Breach Science Publishers, Inc.: Comments in Nuclear and Particle Physics

High Energy Physics Group, University of Hawaii

Institute of Physics Publishing Co., Bristol

Nature Publishing Group, London: Nature

Nuovo Cimento, Italy

Permissions Department, Annual Review Inc.: Annual Reviews in Nucl. and Part. Science

Publication Office: Progress of Theoretical Physics, Kyoto, Japan

Rossiskaja Academija Nauk, Isdatelstwo Nauka, Yadernaja Fizika (Physics of Atomic Nuclei)

Spektrum der Wissenschaft Verlagsgesellschaft mbH, Heidelberg

Springer Verlag GmbH \& Co, KG: Zeitschrift für Physik, Europ. Phys. J.

Wiley-VCH Verlag GmbH, Weinheim: Physik in unserer Zeit, Physik. Blätter

World Scientific Publishing Co Pte Ltd., Singapore: Int. Journal of Modern Physics 
This page is intentionally left blank 


\section{Contents}

Preface

vii

Acknowledgments $\quad$ ix

\section{Chapter 1 Double Beta Decay - Historical Retrospective} and Perspectives 1

1.1 From the Early Days until the Gauge Theory Era . . . . . . . . . . . . . 1

1.1.1 The First Steps in Double Beta Research . . . . . . . . . . . . 1

1.1.2 Double Beta Decay, Gauge Theories and Neutrino Mass . . . . . 10

1.1.2.1 Origins of Neutrino Masses . . . . . . . . . . . . . 10

1.1.2.2 The Double Beta Decay Half Life and the Neutrino Mass 14

1.2 The Nuclear Physics Side - Nuclear Matrix Elements . . . . . . . . . 17

1.2.1 A Breakthrough to the Understanding of the Matrix Elements . 18

1.2.2 QRPA Calculations Including the $p p$-Force $\ldots \ldots \ldots$

1.2.3 Shell Model Calculations . . . . . . . . . . . . . . . . 21

1.2.4 The Operator Expansion Method . . . . . . . . . . . . 22

$1.2 .5 \beta^{+} \beta^{+}, \mathrm{EC} / \mathrm{EC}, \beta^{+} / \mathrm{EC}$ Decay $\ldots \ldots \ldots 23$

1.2.6 Matrix Elements for Exchange of Heavy Particles . . . . . . . . 23

1.3 Double Beta Decay, Neutrino Mass Models and Cosmological Parameters

- Status and Prospects . . . . . . . . . . . . . . . . . . . . 24

1.4 Other Beyond Standard Model Physics: From SUSY and Leptoquarks to Compositeness and Quantum Foam . . . . . . . . . . . . . . 28

1.4 .1 General . . . . . . . . . . . . . . . . . . 28

1.4.2 Doubly Charged Higgs and Pion Double Charge Exchange, and Double Beta Decay . . . . . . . . . . . . . . . . . . . . 29

1.4.3 Superstring-Inspired Models, $R$-Parity Breaking Supersymmetry and Double Beta Decay . . . . . . . . . . . . . . . 29

1.4.4 R-Parity Conserving Supersymmetry and Double Beta Decay . . 30

1.4.5 Leptoquarks and Double Beta Decay . . . . . . . . . . . . . 30

1.4.6 Superheavy Neutrinos and Double Beta Decay . . . . . . . . . 31 
1.4.7 Compositeness and Double Beta Decay . . . . . . . . . . . 32

1.4.8 Sterile Neutrinos, Majorons and Double Beta Decay . . . . . . . 32

1.4.9 Lepton Number Violating Interactions, Nonexponential Decay and Time Dependence of the Weak Interaction . . . . . . . . 33

1.4.10 Test of Lorentz Invariance, Equivalence Principle and Quantum Foam ........................ 33

1.5 The Experimental Race: From the Late Eighties to the Future . . . . . 35

1.5.1 General ...................... 35

1.5.2 Ionisation and Time Projection Chambers, and Combinations with Plastic Scintillators . . . . . . . . . . . . . . . 37

1.5.3 Scintillation Detectors . . . . . . . . . . . . . 41

1.5.4 Semiconductor Detectors ............... 43

1.5.5 Cryogenic Detectors . . . . . . . . . . . . . . 47

1.5.6 Other Large Source Strength Detectors . . . . . . . . . . . . 49

1.6 The Future of Double Beta Decay . . . . . . . . . . . . . . 50

1.6 .1 General ......................... 50

1.6 .2 GENIUS ........................ 54

1.6.3 The Physics Potential of Future Double Beta Decay for Beyond Standard Model Physics . . . . . . . . . . . . . 54

1.7 Conclusion . . . . . . . . . . . . . . . . . . 61

$\begin{array}{ll}\text { Bibliography } & 63\end{array}$

$\begin{array}{lll}\text { Chapter } 2 & \text { Original Articles } & 97\end{array}$

2.1 From the Early Days until the Gauge Theory Era . . . . . . . . 99

2.1.1 The First Steps in Double Beta Research . . . . . . . . . . . . . 99

2.1.1.1 Brief an die Gruppe der Radioaktiven ..., W. Pauli, [Pau30] . . . . . . . . . . . . . . . 101

2.1.1.2 The "Neutrino", H. Bethe and R. Peierls, [Bet34] . . 102

2.1.1.3 Versuch einer Theorie der $\beta$-Strahlen. I, E. Fermi, $[$ Fer34**] . . . . . . . . . . . . . . . 103

2.1.1.4 Double Beta-Disintegration, M. Goeppert-Mayer, [Goe35] . . . . . . . . . . . . . . . . . . 104

2.1.1.5 Teoria Simmetrica dell'Elettrone e del Positrone, $E$. Majorana, $\left[\mathrm{Maj37}^{* *}\right]$. . . . . . . . . . . . 109

2.1.1.6 Sulla Simmetria tra Particelle e Antiparticelle, G. Racah, [Rac37] . . . . . . . . . . . . . . 110

2.1.1.7 On Transition Probabilities in Double Beta-Disintegration, W. H. Furry, [Fur39] . . . . . . . . . . . 117

2.1.1.8 A Measurement of the Half-Life of Double Beta-Decay from ${ }_{50} \mathrm{Sn}^{124}$, E. L. Fireman, [Fir49] . . . . . . . . 127

2.1.1.9 A Re-Investigation of the Double Beta-Decay from $\mathrm{Sn}^{124}$, E. L. Fireman and D. Schwarzer, [Fir52] . . . . . . 128 
2.1.1.10 On the Double Beta-Process, $M$. G. Inghram and J. $H$. Reynolds, [Ing49] . . . . . . . . . . . 131

2.1.1.11 Double Beta-Decay of $\mathrm{Te}^{130}, M$. G. Inghram and J. $H$. Reynolds , [Ing50] . . . . . . . . . . . . 132

2.1.1.12 Half-Life for Double Beta-Decay, C. A. Levine, A. Ghiorso and G. T. Seaborg, [Lev50] . . . . . . . 133

2.1.1.13 The Half-life of ${ }^{130}$ Te Double $\beta$-Decay, $N$. Takaoka and

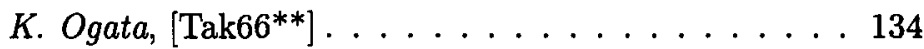

2.1.1.14 Massenspektrometrischer Nachweis von $\beta \beta$-Zerfallsprodukten, T. Kirsten, W. Gentner and O. A. Schaeffer, $\left[\mathrm{Kir} 67^{* *}\right] \ldots \ldots \ldots \ldots \ldots \ldots$

2.1.1.15 Experimental Evidence for the Double-Beta Decay of $\mathrm{Te}^{130}$, T. Kirsten, O. A. Schaeffer, E. Norton and $R$. W. Stoenner, [Kir68] . . . . . . . . . . . 136

2.1.1.16 Geochemical Measurements of Double-Beta Decay, $O$. K. Manuel, [Man91] . . . . . . . . . . . . . . 140

2.1.1.17 Neutrino Mass Limits from a Precise Determination of $\beta \beta$-Decay Rates of ${ }^{128} \mathrm{Te}$ and ${ }^{130} \mathrm{Te}, T$. Bernatowicz, $J$. Brannon, R. Brazzle, R. Cowsik, C. Hohenberg and F. Podosek, [Ber92] . . . . . . . . . . . . . 149

2.1.1.18 Precise Determination of Relative and Absolute $\beta \beta$ Decay Rates of ${ }^{128} \mathrm{Te}$ and ${ }^{130} \mathrm{Te}, T$. Bernatowicz, $J$. Brannon, R. Brazzle, R. Cowsik, C. Hohenberg and F.

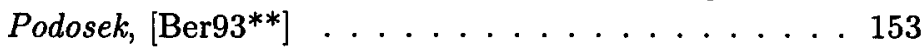

2.1.1.19 Half-Life of ${ }^{130}$ Te Double- $\beta$ Decay Measured with Geologically Qualified Samples, N. Takaoka, Y. Motomura and K. Nagao, [Tak96**] . . . . . . . . . . . . 154

2.1.1.20 Study of the Double Beta Decay of ${ }^{130} \mathrm{Te}, Y u . G$. Zdesenko, I. A. Mytsyk, A. S. Nikolaiko and V. N. Kuts, $\left[\mathrm{Zde} 80 \mathrm{a}^{* *}\right] \ldots \ldots \ldots \ldots \ldots \ldots$

2.1.1.21 Double Beta Decay of ${ }^{238} \mathrm{U}$, A. L. Turkevich, T. E. Economou and G. A. Cowan, [Tur91] . . . . . . 156

2.1.1.22 Limits for Lepton-Conserving and Lepton-Nonconserving Double Beta Decay in $\mathrm{Ca}^{\mathbf{4 8}}, E$. der Mateosian and M. Goldhaber, [Mat66] . . . . . . . . . . . 160

2.1.1.23 Double $\beta$ Decay and Conservation of Lepton Charge, Yu. G. Zdesenko, [Zde80**] . . . . . . . . . 166

2.1.2 Double Beta Decay, Gauge Theories and Neutrino Mass: Origins of Neutrino Masses . . . . . . . . . . . . . . . . . 167

2.1.2.1 Horizontal Gauge Symmetry and Masses of Neutrinos, T. Yanagida, [Yan79] . . . . . . . . . . . . 169 
2.1.2.2 Massive Neutrinos in Gauge Theories, $P$. Langacker, [Lan88] . . . . . . . . . . . . . . . 173

2.1.2.3 Neutrino Mass Textures and the Nature of New Physics Implied by Present Neutrino Data, R. N. Mohapatra, [Moh97] . . . . . . . . . . . . . . . . . 218

2.1.2.4 Neutrino Mass and Spontaneous Parity Nonconservation, R. N. Mohapatra and G. Senjanović, [Moh80] . . 231

2.1.2.5 Neutrino Mass and Baryon-Number Nonconservation in Superstring Models, R. N. Mohapatra and J.W.F. Valle, [Moh86b] . . . . . . . . . . . . 235

2.1.2.6 Neutrinos in Left-Right Symmetric, $\mathrm{SO}(10)$ and Superstring Inspired Models, R. N. Mohapatra, [Moh88a] . . 239

2.1.2.7 Neutrino Textures in the Light of SUPERKAMIOKANDE Data and a Realistic String Model, J. Ellis, G. K. Leontaris, S. Lola and D. V. Nanopoulos, [El198**] . . 269

2.1.2.8 Zee Neutrino Mass Model in a SUSY Framework, $K$. Cheung and Otto C. W. Kong, [Che2000**] . . . . . 270

2.1.2.9 Fermion Masses and Neutrino Oscillations in $\mathrm{SO}(10)$ Supersymmetric Grand Unified Theory with $D_{3} \times$ U(1) Family Symmetry, R. Dermišsek and S. Raby, $[$ Der2000**] . . . . . . . . . . . . . 271

2.1.2.10 Neutrino Oscillations in a predictive SUSY GUT, $T$. Blažek, S. Raby and K. Tobe, [Bla99**] . . . . . . . . 272

2.1.2.11 Neutrino Masses within the Minimal Supersymmetric Standard Model, M. Cvetič and P. Langacker, [Cve92a] . . . . . . . . . . . . . 273

2.1.2.12 New Directions for New Dimensions: From Strings to Neutrinos to Axions to ..., K. R. Dienes, [Die2000**]

2.1.2.13 Reconciling Present Neutrino Puzzles: Sterile Neutrinos as Mirror Neutrinos, Z. G. Berezhiani and R. N. Mohapatra, [Ber95] . . . . . . . . . . . 279

2.1.2.14 Exotic Mechanisms for Neutrino Masses, Z. Berezhiani, $[$ Ber99a**] . . . . . . . . . . . . . . . 284

2.1.2.15 Neutrino Masses in $\mathrm{SU}(2) \otimes \mathrm{U}(1)$ Theories, J. Schechter and J. W. F. Valle, $\left[\mathrm{Sch} 80^{* *}\right]$. . . . . . . . . 285

2.1.2.16 Unification and Supersymmetry, R. N. Mohapatra, [Moh $\left.86 \mathrm{c}^{* *}\right] \ldots \ldots \ldots \ldots \ldots \ldots . \ldots \ldots$

2.1.2.17 On Seeing the First Double Beta Decay, S. P. Rosen, $[$ Ros88**] . . . . . . . . . . . . . . . 287

2.1.2.18 The Physics of Massive Neutrinos, B. Kayser, [Kay89**] 288

2.1.2.19 Neutrinoless Double- $\beta$ Decay in SU(2) $\times \mathrm{U}(1)$ Theories, J. Schechter and J. W. F. Valle, [Sch82a] . . . . 289 
2.1.2.20 Neutrinoless Double $\beta$ Decay with Quasi-Dirac Neutrinos, J. W. F. Valle, [Val83] . . . . . . . . 293

2.1.3 Double Beta Decay, Gauge Theories and Neutrino Mass: The Double Beta Decay Half Life and the Neutrino Mass . . . . . . . . . . . 297

2.1.3.1 Neutrino Mass, the Right-Handed Interaction and the Double Beta Decay: I, M. Doi, T. Kotani, H. Nishiura, K. Okuda and E. Takasugi, [Doi81a] . . . . . . . 299

2.1.3.2 Neutrino Mass, the Right-Handed Interaction and the Double Beta Decay: II, M. Doi, T. Kotani, H. Nishiura, K. Okuda and E. Takasugi, [Doi81b**] . . . . . . . . 325

2.1.3.3 Double Beta Decay, M. Doi, T. Kotani, H. Nishiura and E. Takasugi, [Doi83**] . . . . . . . . . . 326

2.1.3.4 The Energy Spectrum and the Angular Correlation in the $\beta \beta$ Decay, M. Doi, T. Kotani, H. Nishiura and E. Takasugi, [Doi83b**] . . . . . . . . . . 327

2.1.3.5 Double Beta Decay and Majorana Neutrino, M. Doi, T. Kotani and E. Takasugi, [Doi85**] . . . . . . . 328

2.1.3.6 Neutrinoless Double Beta-Decay with Majoron Emission, M. Doi, T. Kotani and E. Takasugi, [Doi88**] . . 329

2.1.3.7 Neutrinoless Modes of Double Beta Decay, M. Doi and T. Kotani, [Doi93**] . . . . . . . . . . . . . 330

2.1.3.8 Double Beta Decay, W. Haxton, $\left[\operatorname{Hax} 84^{* *}\right]$. . . . . . . 331

2.1.3.9 Are Massive Neutrinos Majorana Particles?, T. Kotani,

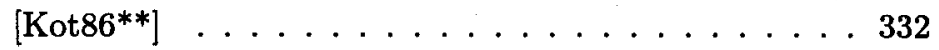

2.1.3.10 Double Beta Decay, Neutrino Mass and Nuclear Structure, K. Muto and H. V. Klapdor, [Mut88] . . . . . 333

2.1.3.11 Double Beta Decay, T. Tomoda, [Tom91**] . . . . . . . 388

2.1.3.12 The Weak Interaction in Nuclear, Particle and Astrophysics, K. Grotz and H. V. Klapdor, [Gro89/90**] . . 389

2.1.3.13 Double Beta Decay, H. Primakoff and S. P. Rosen, $[$ Pri59**] . . . . . . . . . . . . . 390

2.1.3.14 Lepton Conservation and Double Beta-Decay, $E$. Greuling and R. C. Whitten, [Gre60**] . . . . . . . . 391

2.1.3.15 Baryon Number and Lepton Number Conservation Laws, H. Primakoff and S. P. Rosen, [Pri81**] . . . . 392

2.1.3.16 Towards a Superformula for Neutrinoless Double Beta Decay, H. Päs, M. Hirsch, H. V. Klapdor-Kleingrothaus and S. G. Kovalenko, [Pae99] . . . . . . . . . 393

2.1.3.17 A Superformula for Neutrinoless Double Beta Decay II: The Short Range Part, H. Päs, M. Hirsch, H. V. Klapdor-Kleingrothaus and S. G. Kovalenko, $[\mathrm{Pae2000**}] \ldots \ldots \ldots \ldots . \ldots . \ldots . \ldots 39$ 
2.1.3.18 Lepton-Number Conservation and the Double- $\beta$ Decay of ${ }^{128} \mathrm{Te}$ and ${ }^{130} \mathrm{Te}, W$. C. Haxton, G. J. Stephenson and D. Strottman, [Hax82a] . . . . . . . . . . 399

2.1.3.19 Nuclear Double-Beta Decay and a New Limit on Lepton Nonconservation, $H$. Primakoff and $S$. P. Rosen, [Pri69] . . . . . . . . . . . . . . 409

2.1.3.20 Double Beta Decay and the Majorana Mass of the Electron Neutrino, W. C. Haxton, G. J. Stephenson and D. Strottman, [Hax81] . . . . . . . . . . . 418

2.1.3.21 Rejection of Evidence for Nonzero Neutrino Rest Mass from Double Beta Decay, T. Kirsten, H. Richter and E. Jessberger, [Kir83] . . . . . . . . . . . . . 422

2.2 The Nuclear Physics Side - Nuclear Matrix Elements . . . . 427

2.2.1 A Breakthrough to the Understanding of the Matrix Elements . . . 427

2.2.1.1 Double Beta Decay of ${ }^{128} \mathrm{Te} /{ }^{130} \mathrm{Te}$ and the Question of a Neutrino Majorana Mass, K. Grotz, H. V. Klapdor and J. Metzinger, [Gro83a] . . . . . . . . . . . .

2.2.1.2 The Quenching of low-Energetic Nuclear Gamow-Teller Strength by $\Delta$-Excitations, $K$. Grotz, H. V. Klapdor and J. Metzinger, [Gro83c] . . . . . . . . . . . 434

2.2.1.3 Calculation of Double Beta Decay of ${ }^{76} \mathrm{Ge},{ }^{82} \mathrm{Se},{ }^{128,130} \mathrm{Te}$, H. V. Klapdor and K. Grotz, [Kla84] . . . . . . . . . 439

2.2.1.4 The Neutrino Mass from Double Beta Decay, K. Grotz and H. V. Klapdor, [Gro85b] . . . . . . . . . . 445

2.2.1.5 Predictions of $2 \nu$ and $0 \nu$ Double Beta Decay Rates for Nuclei with $A \geq 70, K$. Grotz and H.V. Klapdor, [Gro85a] . . . . . . . . . . . . . 449

2.2.1.6 Calculation of Double Beta Decay Rates and the Neutrino Mass, K. Grotz and H. V. Klapdor, [Gro86a**] . 454

2.2.2 QRPA Calculations Including the $p p$-Force . . . . . . . . . . 455

2.2.2.1 Suppression of the Two-Neutrino Double-Beta Decay by Nuclear-Structure Effects, $P$. Vogel and $M$. R. Zirnbauer, $[\operatorname{Vog} 86] \ldots \ldots$. . . . . . . . . . . 457

2.2.2.2 Nuclear Structure Effects in Double-Beta Decay, $J$. Engel, P. Vogel and M. R. Zirnbauer, [Eng88**] . . . 461

2.2.2.3 Effects of Ground-State Correlations on $2 \nu \beta \beta$ Decay Rates and Limitations of the QRPA Approach, $K$. Muto, E. Bender and H. V. Klapdor, [Mut89**] . . . . . . 462

2.2.2.4 Nuclear Structure Effects on the Neutrinoless Double Beta Decay, K. Muto, E. Bender and H. V. Klapdor, [Mut89a] ................. 463 
2.2.2.5 Calculation of $2 \nu$ and $0 \nu$ Double-Beta Decay Rates, A. Staudt, K. Muto and H. V. Klapdor-Kleingrothaus,

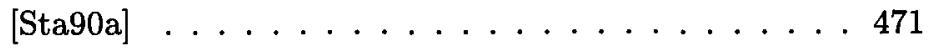

2.2.2.6 $\beta \beta$ Decay of ${ }^{76} \mathrm{Ge}$ with Renormalized Effective Interaction Derived from Paris, Bonn and Reid Potentials, $A$. Staudt, T. T. S. Kuo and H. V. Klapdor-Kleingrothaus, [Sta90] . . . . . . . . . . . . . 4 477

2.2.2.7 $\beta \beta$ Decay of ${ }^{128} \mathrm{Te},{ }^{130} \mathrm{Te}$, and ${ }^{76} \mathrm{Ge}$ with Renormalized Effective Interactions Derived from Paris and Bonn Potentials, A. Staudt, T. T. S. Kuo and H. V. KlapdorKleingrothaus, $[$ Sta92**] . . . . . . . . . . . . 484

2.2.2.8 Nonaccelerator Particle Physics, H. V. Klapdor-Kleingrothaus and A. Staudt, [Kla95/98**] . . . . . . . . . 485

2.2.2.9 Renormalized Proton-Neutron Quasiparticle RandomPhase Approximation and Its Application to Double Beta Decay, J. Toivanen and J. Suhonen, [Toi95] . . . 486

2.2.2.10 Gamow-Teller Matrix Elements for Two-Neutrino Double Beta Decay within a Second Quasi-RandomPhase Approximation, S. Stoica and W. A. Kaminski, [Sto93a] . . . . . . . . . . . . . . 490

2.2.2.11 Second-QRPA Calculations for Two Neutrino DoubleBeta Decay, S. Stoica, [Sto96**] . . . . . . . . . . 493

2.2.2.12 Double-Beta Decay Matrix Elements for ${ }^{76} \mathrm{Ge}, S$. Stoica and H. V. Klapdor-Kleingrothaus, [Sto2000**] 494

2.2.2.13 QRPA and RQRPA Calculations of Neutrinoless Double-Beta Decay beyond the Point of Collapse, $G$. Pantis, F. Šmkovic and A. Faessler, [Pan98a**] . . . . 495

2.2.2.14 Double Beta Decay, A. Faessler and F. Šmkovic, $[$ Fae98**] . . . . . . . . . . . . . . . 496

2.2.2.15 A Field Theory Approach to Two-Neutrino Double Beta Decay, F. Šmkovic and G. Pantis, [Sim99a**] . . . . 497

2.2.3 Shell Model Calculations ... . . . . . . . . . . . . . . . 499

2.2.3.1 Shell Model Studies of the Double Beta Decays of ${ }^{76} \mathrm{Ge}$, ${ }^{82} \mathrm{Se}$ and ${ }^{136} \mathrm{Xe}$, E. Caurier, F. Nowacki, A. Poves and J. Retamosa, [Cau96] . . . . . . . . . . . . . 501

2.2.3.2 Shell Model Calculation for Two-Neutrino Double Beta Decay of ${ }^{48} \mathrm{Ca}, L$. Zhao, B. A. Brown and W. A. Richter, $[$ Zha90**] . . . . . . . . . . . . . 505

2.2.3.3 Shell Model Monte Carlo Method for Two-Neutrino Double Beta Decay, P. B. Radha, D. J. Dean, S. E. Koonin, T. T. S. Kuo, K. Langanke, A. Poves, J. Retamosa and P. Vogel, [Rad96] . . . . . . . . 506 
2.2.3.4 A Comparative Study of Double Beta Decay by Shell Model and Quasiparticle RPA, K. Muto, E. Bender and H. V. Klapdor-Kleingrothaus, [Mut91] . . . . . . . . 510

2.2.4 The Operator Expansion Method . . . . . . . . . . . . . . . . 521

2.2.4.1 An Alternative Approach in Nuclear Double Beta Decay Theory, C. R. Ching and T. H. Ho, [Chi88] . . 523

2.2.4.2 A Discussion of the Question in Connecxion with the Expansion of a Divergent Series Encountered in Calculating Nuclear $2 \nu-2 \beta$ Decay Matrix Element, $C$. $R$. Ching and T. H. Ho, [Chi89] . . . . . . . . . 530

2.2.4.3 A New Method of the Calculation of the TwoNeutrino Double Beta Decay Amplitudes, F. Šimkovic, $\left[\operatorname{Sim} 89^{* *}\right] \ldots \ldots \ldots \ldots \ldots \ldots \ldots$

2.2.4.4 New Developments in the Theory of Double $\beta$ Decay, M. Gmitro and F. Šimkovic, [Gmi90] . . . . . . . . 534

2.2.4.5 Two-Neutrino Double Beta Decay with Operator Expansion Method, $X . R$. Wu, A. Staudt, H. V. KlapdorKleingrothaus, Cheng-Rui Ching and Tso-Hsiu Ho, [Wu91] . . . . . . . . . . . . . . . 541

2.2.4.6 Tensor Force and Operator Expansion Method for Nuclear Double Beta Decay, X. R. Wu, A. Staudt, T. T. S. Kuo and H. V. Klapdor-Kleingrothaus, [Wu92]

2.2.4.7 New Theoretical Results of $2 \nu \beta \beta$ Decay with the Operator Expansion Method, X. R. Wu, M. Hirsch, A. Staudt, H. V. Klapdor-Kleingrothaus, Cheng-Rui Ching and Tso-Hsiu Ho, [Wu93]

2.2.4.8 Matrix Elements for $0 \nu \beta \beta$ Decay Calculated with the Operator Expansion Method and QRPA Wave Functions, $M$. Hirsch, $X . R$. Wu, H. V. KlapdorKleingrothaus, Ching Cheng-Rui and Ho Tso-Hsiu, $[$ Hir93**] . . . . . . . . . . . . . . . . 558

2.2.4.9 Operator Expansion Method and Nuclear $\beta \beta$ Decay, M. Hirsch, X. R. Wu, H. V. Klapdor-Kleingrothaus, Ching Cheng-Rui and Ho Tso-Hsiu, [Hir94b**] . . . 559

2.2.4.10 Extended Operator Expansion Method for Neutrinoless Double Beta Decay, M. Hirsch, O. Kadowaki, H. V. Klapdor-Kleingrothaus, K. Muto and T. Oda, [Hir95a] . . . . . . . . . . . . 560

2.2.4.11 Two-Neutrino Double Beta Decay: Critical Analysis, F. Simkovic, G. Pantis and A. Faessler, [Sim98**] . . 573 
$2.2 .5 \beta^{+} \beta^{+}, \mathbf{E C} / \mathbf{E C}, \beta^{+} /$EC Decay . . . . . . . . . . 575

2.2.5.1 Nuclear Matrix Elements for Double Positron Emission, A. Staudt, K. Muto and H. V. Klapdor-Kleingrothaus, $[$ Sta91] . . . . . . . . . . . 577

2.2.5.2 Nuclear Structure Calculation of $\beta^{+} \beta^{+}, \beta^{+} / \mathrm{EC}$ and EC/EC Decay Matrix Elements, M. Hirsch, K. Muto, T. Oda and H. V. Klapdor-Kleingrothaus, [Hir94] . . . 582

2.2.6 Matrix Elements for Exchange of Heavy Particles . . . . 593

2.2.6.1 $e^{-} e^{-} \longrightarrow W^{-} W^{-}-$Mediated by Heavy Neutrino Flavors, P. Minkowski, [Min96**] . . . . . . . . . 595

2.2.6.2 Heavy Majorana Neutrinos in $e^{-} e^{-}$Collisions, C. Greub and P. Minkowski, [Gre96**] . . . . . . . . . . 596

2.2.6.3 On Nuclear Matrix Element Uncertainties in Short Range $0 \nu \beta \beta$ Decay, H. V. Klapdor-Kleingrothaus and H. Päs, [Kla2000g] . . . . . . . . . . . . 597

2.3 Effective Neutrino Masses from Double Beta Decay, Neutrino Mass Models and Cosmological Parameters - Present Status and Prospects . . . . . . . . . . . . . . . . . . 6 601

2.3.0.1 Neutrino Mass Explanations of Solar and Atmospheric Neutrino Deficits and Hot Dark Matter, D. O. Caldwell and R. N. Mohapatra, [Cal93] . . . . . . . . . . 603

2.3.0.2 Neutrino Masses and Oscillations, A. Yu. Smirnov,

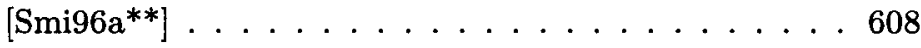

2.3.0.3 The HEIDELBERG-MOSCOW Double Beta Experiment with Enriched ${ }^{76} \mathrm{Ge}$ : Status and Perspectives, $H$. V. Klapdor-Kleingrothaus, $\left[\mathrm{Kla93}^{* *}\right]$. . . . . . . 609

2.3.0.4 Accommodating Solar and Atmospheric Neutrino Deficits, Hot Dark Matter, and a double $\beta$ Decay Signal, D. O. Caldwell and R. N. Mohapatra, [Cal94**] . . . 610

2.3.0.5 Perspectives of Double Beta and Dark Matter Search as Windows to New Physics, H. V. Klapdor-Kleingrothaus, $\left[\mathrm{Kla}^{2} 9 \mathrm{e}^{* *}\right] \ldots \ldots \ldots 11$

2.3.0.6 Limits on the Majorana Neutrino Mass in the $0.1 \mathrm{eV}$ Range, HEIDELBERG-MOSCOW Collaboration, $L$. $B a$ udis, A. Dietz, G. Heusser, H. V. Klapdor-Kleingrothaus, I. V. Krivosheina, S. Kolb, B. Majorovits, V.F. Melnikov, H. Päs, F. Schwamm, H. Strecker, V. Alexeev, A. Balysh, A. Bakalyarov, S. T. Belyaev, V. I. Lebedev and $S$. Zhukov, [HM99] . . . . . . . 612 
2.3.0.7 Double Beta Decay of ${ }^{76} \mathrm{Ge}$ : New Results from the HEIDELBERG-MOSCOW Experiment, HEIDELBERGMOSCOW Collaboration, $H$. V. Klapdor-Kleingrothaus, L. Baudis, A. Dietz, I. V. Krivosheina, G. Heusser, St. Kolb, B. Majorovits, H. Päs, H. Strecker, H. Tu, V. Alexeev, A. Balysh, A. Bakalyarov, S. T. Belyaev, V. I. Lebedev and S. Zhukov, [HM2000**] . . . . . . 616

2.3.0.8 Constraints on Mixing Angles of Majorana Neutrinos, R. Adhikari and G. Rajasekaran, [Adh98] . . . . . . 617

2.3.0.9 Constraining Almost Degenerate Three-Flavor Neutrinos, H. Minakata and O. Yasuda, [Min97a**] . . . . 622

2.3.0.10 Constraining Degenerate Neutrino Mass Model and Implications, O. Yasuda, [Yas99] . . . . . . . . . . 623

2.3.0.11 Answering the Sphinx's Questions on Neutrinos, $H$. Minakata, $\left[\operatorname{Min} 2000^{* *}\right] \ldots \ldots 636$

2.3.0.12 Neutrinos on Earth and in the Heavens, H. Georgi and S. L. Glashow, [Geo2000] . . . . . . . . . . . 637

2.3.0.13 Simple Connection between Atmospheric and Solar Neutrino Vacuum Oscillations, E. Ma, [Ma99] . . . . 641

2.3.0.14 Neutrinoless Double- $\beta$ Decay with Three or Four Neutrino Mixing, C. Giunti, [Giu99] . . . . . . . . . . . 645

2.3.0.15 Neutrino Mass Spectrum and Neutrinoless Double Beta Decay, H. V. Klapdor-Kleingrothaus, H. Päs and A. Yu. Smirnov, [Kla2000] . . . . . . . . . . 653

2.3.0.16 Signal of Neutrinoless Double Beta Decay, Neutrino Spectrum and Oscillation Scenarios, F. Vissani, [Vis99] 700

2.3.0.17 Neutrinoless Double Beta Decay in Four-Neutrino Models, A. Kalliomäki and J. Maalampi, [Kal2000**] . . . 715

2.3.0.18 The Quest for the Neutrino Mass Spectrum, H. V. Klapdor-Kleingrothaus and H. Päs, [Kla2000i**] . . . 716

2.3.0.19 New Limits on Dark-Matter Wearkly Interacting Particles from the Heidelberg-Moscow Experiment, HEIDELBERG-MOSCOW Collaboration, L. Baudis, J. Hellmig, G. Heusser, H. V. Klapdor-Kleingrothaus, $S$. Kolb, B. Majorovits, H. Päs, Y. Ramachers, H. Strecker, V. Alexeev, A. Balysh, A. Bakalyarov, S. T. Belyaev, V. I. Lebedev and S. Zhukov, [HM98**] . . . . . . . . 717

2.4 Other Beyond Standard Model Physics: From SUSY and Leptoquarks to Compositeness and Quantum Foam . . . . . . . . 719 2.4 .1 General . . . . . . . . . . . . . . . . . . . . 719

2.4.1.1 Neutrinoless Double Beta Decay and Physics Beyond the Standard Model, R. N. Mohapatra, [Moh96] . . . 721 
2.4.1.2 Double Beta Decay - Physics Beyond the Standard Model, H. V. Klapdor-Kleingrothaus, [Kla96b**] . . . . 746

2.4.1.3 Doppelbetazerfall — Physik jenseits des Standardmodells, H. V. Klapdor-Kleingrothaus, [Kla98b] . . . . 747

2.4.1.4 Neutrinoless Double Beta Decay and New Physics in the Neutrino Sector, H. V. Klapdor-Kleingrothaus and H. Päs, [Kla2000e] . . . . . . . . . . . . 755

2.4.1.5 Massive Neutrinos in Physics and Astrophysics, R. N. Mohapatra and P. B. Pal, [Moh91**] . . . . . . . 763

2.4.1.6 Particle Astrophysics, H. V. Klapdor-Kleingrothaus and K. Zuber, [Kla97/2000**] . . . . . . . . . . . 764

2.4.2 Doubly Charged Higgs and Pion Double Charge Exchange, and Double Beta Decay . . . . . . . . . . . . . . . 765

2.4.2.1 New Contribution to Neutrinoless Double Beta Decay in Gauge Models, R. N. Mohapatra and J. D. Vergados, [Moh81] ................. 767

2.4.2.2 Pion-Double-Charge-Exchange Conribution to Neutrinoless Double- $\beta$ Decay, J. D. Vergados, [Ver82] . . . . 771

2.4.3 Superstring-Inspired Models, $R$-Parity Breaking Supersymmetry and Double Beta Decay . . . . . . . . . . . . . . . 775

2.4.3.1 Lepton Phenomenology and Neutrinoless Double $\beta$ Decay in Superstring Inspired Theories, G. K. Leontaris and J. D. Vergados, [Leo89**] . . . . . . . . 777

2.4.3.2 New Contributions to Neutrinoless Double-Beta Decay in Supersymmetric Theories, R. N. Mohapatra, [Moh86a] . . . . . . . . . . . . . 778

2.4.3.3 New Constraints on $R$-Parity-Broken Supersymmetry from Neutrinoless Double Beta Decay, M. Hirsch, H. V. Klapdor-Kleingrothaus and S. G. Kovalenko, $[$ Hir95**] . . . . . . . . . . . . . 783

2.4.3.4 New Contributions to Supersymmetric Mechanism of Neutrinoless Double Beta Decay, M. Hirsch, $H$. $V$. Klapdor-Kleingrothaus and S. G. Kovalenko, $[$ Hir95b**] . . . . . . . . . . . . . . . 784

2.4.3.5 Test von Supersymmetrie: Doppelbetazerfall und Teilchenbeschleuniger, M. Hirsch, H. V. Klapdor-Kleingrothaus, $[\mathrm{Hir} 95 \mathrm{c}] \ldots \ldots . \ldots . . . . . .785$

2.4.3.6 Supersymmetry and Neutrinoless Double Beta Decay, $M$. Hirsch, H. V. Klapdor-Kleingrothaus and $S$. G. Kovalenko, [Hir96] . . . . . . . . . . . . 787 
2.4.3.7 Leptoquark/Squark Interpretation of HERA Events: Virtual Effects in $e^{+} e^{-}$Annihilation to Hadrons, $J$. Kalinowski, R. Rückl, H. Spiesberger and P. M. Zerwas, $\left[\mathrm{Kal97}^{* *}\right] \ldots \ldots \ldots \ldots$. . . . . . . 805

2.4.3.8 High $Q^{2}$ DIS at HERA and Squark Production, $H$. Dreiner, M. Krämer and P. Morawitz, [Dre98**] . . 806

2.4.3.9 Grand Unified Theory Constrained Supersymmetry and Neutrinoless Double $\beta$ decay, $A$. Wodecki $W . A$. Kaminski and F. Šimkovic, [Wod99a**] . . . . . . . . 807

2.4.3.10 Limits on $R$-Parity Nonconservation from the Neutrinoless Double Beta Decay in the MSSM with Gauge Mediated Breaking, A. Wodecki and W. A. Kaminiski, [Wod99] . . . . . . . . . . . . . . 808

2.4.3.11 New Vector-Scalar Contributions to Neutrinoless Double Beta Decay and Constraints on $R$-Parity Violation, K. S. Babu and R. N. Mohapatra, [Bab95] . . . . . . 813

2.4.3.12 On the SUSY Accompanied Neutrino Exchange Mechanism of Neutrinoless Double Beta Decay, M. Hirsch, H. V. Klapdor-Kleingrothaus and S. G. Kovalenko, [Hir96a] ..................... 817

2.4.3.13 Improved Bounds on SUSY Accompanied Neutrinoless Double Beta Decay, H. Päs, M. Hirsch, H. V. KlapdorKleingrothaus, $[\mathrm{Pae} 99 \mathrm{a}]$. . . . . . . . . . 824

2.4.4 R-Parity Conserving Supersymmetry and Double Beta Decay . . . 829

2.4.4.1 B-L-Violating Masses in Softly Broken Supersymmetry, M. Hirsch, H. V. Klapdor-Kleingrothaus and $S$. G. Kovalenko, [Hir97a**] . . . . . . . . . 831

2.4.4.2 Sneutrino Oscillations and Neutrinoless Double Beta Decay, M. Hirsch, H. V. Klapdor-Kleingrothaus and $S$. G. Kovalenko, $\left[\mathrm{Hir} 97 \mathrm{~b}^{* *}\right]$. . . . . . . . . 832

2.4.4.3 R-Parity-Conserving Supersymmetry, Neutrino Mass, and Neutrinoless Double Beta Decay, M. Hirsch, $H$. V. Klapdor-Kleingrothaus and S. G. Kovalenko, [Hir98a]

2.4.4.4 Phenomenological Implications of "Majorana" Sneutrinos at Future Accelerators, $M$. Hirsch, H. V. KlapdorKleingrothaus, St. Kolb and S. G. Kovalenko, [Hir98d]

2.4.4.5 Light Lepton Number Violating Sneutrinos and the Baryon Number of the Universe, H. V. Klapdor-Kleingrothaus, St. Kolb and V. A. Kuzmin, [Kla2000h**] . . 852 
2.4.5 Leptoquarks and Double Beta Decay . . . . . . . . . . . . . . . 853

2.4.5.1 New Leptoquark Mechanism of Neutrinoless Double $\beta$ Decay, M. Hirsch, H. V. Klapdor-Kleingrothaus and $S$. G. Kovalenko, [Hir96d] . . . . . . . . . . 855

2.4.5.2 New Low-Energy Leptoquark Interactions, M. Hirsch, H. V. Klapdor-Kleingrothaus and S. G. Kovalenko, [Hir96c] . . . . . . . . . . . . . . . . 859

2.4.5.3 Neutrinoless Double Beta Decay and the HERA "Anomaly", M. Hirsch, H. V. Klapdor-Kleingrothaus and S. G. Kovalenko, [Hir98c] . . . . . . . . 865

2.4.5.4 Much Ado About Leptoquarks: A Comprehensive Analysis, J. L. Hewett and T. G. Rizzo, [Hew97**] . . 874

2.4.6 Superheavy Neutrinos and Double Beta Decay. . . . . . . . . . 875

2.4.6.1 Limits on the Mass of the Right-Handed Majorana Neutrino, R. N. Mohapatra, [Moh86] _. . . . . . 877

2.4.6.2 Double Beta Decay in Left-Right Symmetric Models, M. Hirsch, H. V. Klapdor-Kleingrothaus and $O$. $P a$ nella, [Hir96b] . . . . . . . . . . . 879

2.4.6.3 Inverse Neutrinoless Double Beta Decay and $\Delta L=2$ Processes at Linear Colliders, G. Bélanger, [Bel98] . . 885

2.4.7 Compositeness and Double Beta Decay . . . . . . . . . . . 901

2.4.7.1 Neutrinoless Double $\beta$ Decay with Composite Neutrinos, O. Panella, C. Carimalo, Y. N. Srivastava and A. Widom, $\left[\mathrm{Pan} 97^{* *}\right] \ldots \ldots . . . . . . . .903$

2.4.7.2 Production of Like Sign Dileptons in $p-p$ Collisions through Composite Majorana Neutrinos, O. Panella, C. Carimalo and Y. N. Srivastava, [Pan2000] . . . . 904

2.4.8 Sterile Neutrinos, Majorons and Double Beta Decay . . . . . . . . . 919

2.4.8.1 Spontaneously Broken Lepton Number and Cosmological Constraints on the Neutrino Mass Spectrum, Y. Chikashige, R. N. Mohapatra and R. D. Peccei, [Chi80] . . . . . . . . . . . . . . 921

2.4.8.2 New Class of Majoron-Emitting Double- $\beta$ Decays, $C$. P. Burgess and J. M. Cline, [Bur93**] . . . . . . . 925

2.4.8.3 Scalar-Emitting Modes in Double-Beta Decay, C. $P$. Burgess, $[$ Bur95**] . . . . . . . . . . . . 926

2.4.8.4 Limit on Neutrinoless Double-Beta Decay with Majoron Emission in ${ }^{82} \mathrm{Se}, S$. R. Elliot, A. A. Hahn, M. K. Moe, [Ell87] . . . . . . . . . . 927 
2.4.8.5 Investigation of the Majoron-Accompanied DoubleBeta Decay Mode of ${ }^{76} \mathrm{Ge}, M$. Beck, F. Bensch, J. Bockholt, G. Heusser, M. Hirsch, H. V. Klapdor-Kleingrothaus, B. Maier, F. Petry, A. Piepke, H. Strecker, M. Völlinger, K. Zuber, A. Balysh, S. T. Belyaev, A. Demehin, A. Gurov, I. Kondratenko, V. I. Lebedev and A. Müller, [Bec93] . . . . . . . . . . . . . 930

2.4.8.6 On the Observability of Majoron Emitting Double Beta Decays, M. Hirsch, H. V. Klapdor-Kleingrothaus, S. G. Kovalenko and H. Päs, [Hir96e] . . . . . . . . 933

2.4.8.7 Bounds on New Majoron Models from the HEIDELBERG-MOSCOW Experiment, M. Günther, J. Hellmig, G. Heusser, M. Hirsch, H. V. Klapdor-Kleingrothaus, B. Maier, H. Päs, F. Petry, Y. Ramachers, H. Strecker, M. Völlinger, A. Balysh, S. T. Belyaev, A. Demehin, A. Gurov, I. Kodratenko, D. Kotel'nikov, V. I. Lebedev and A. Müller, [Gun96**] . . . . . . . . . 940

2.4.9 Lepton Number Violating Interactions, Nonexponential Decay and Time Dependence of the Weak Interaction . . . . . . . . . . . . . 941

2.4.9.1 Lepton Number Violation Interactions and their Effects on Neutrino Oscillation Experiments, S. Bergmann, $H$. V. Klapdor-Kleingrothaus and H. Päs, [Ber2000**] . . 943

2.4.9.2 Time Scale of Short Time Deviations from Exponential Decay, K. Grotz and H. V. Klapdor, [Gro84] . . . . . . 944

2.4.9.3 Is the Weak Interaction Constant Really Constant?, $A$. S. Barabash, [Bar99**] . . . . . . . . . . . . 947

2.4.10 Test of Lorentz Invariance, Equivalence Principle and Quantum Foam . . . . . . . . . . . . . . . . . 949

2.4.10.1 Test of Special Relativity and Equivalence Principle from Neutrinoless Double Beta Decay, $H$. V. KlapdorKleingrothaus, H. Päs and U. Sarkar, [Kla99c] . . . . . 951

2.4.10.2 Effects of New Gravitational Interactions on Neutrinoless Double Beta Decay, H. V. Klapdor-Kleingrothaus, H. Päs and U. Sarkar, [Kla2000a] . . . . . . . . . 955

2.4.10.3 Comment on "Closing the Neutrinoless Double Beta Decay Window into VEP and/or VLI, $H$. V. KlapdorKleingrothaus, H. Päs and U. Sarkar, [Kla2000f] . . . 961

2.4.10.4 Effect of Violation of Quantum Mechanics on Neutrino Oscillation, Y. Liu, L.-Z. Hu and M.-L. Ge, [Liu97**] 965

2.4.10.5 Remarks on Neutrino Tests of Special Relativity, S. $L$. Glashow, A. Halprin, P. I. Krastev, C. N. Leung and J. Panteleone, [Gla97] 
2.4.10.6 Effects of Quantum Space Time Foam in the Neutrino Sector, H. V. Klapdor-Kleingrothaus, H. Päs and $U$. Sarkar, [Kla2000b] . . . . . . . . . . . . 968

2.5 The Experimental Race: from the Late Eighties to the Future 975

2.5 .1 General . . . . . . . . . . . . . . . . . 975

2.5.1.1 Weak and Electromagnetic Interactions in Nuclei, $H$. V. Klapdor, [Kla86b**] . . . . . . . . . . . . . 977

2.5.1.2 Neutrinos, H. V. Klapdor, [Kla88**]. . . . . . . . 978

2.5.1.3 Neutrino Physics, H. V. Klapdor-Kleingrothaus and $B$. Povh, [Kla88a**] . . . . . . . . . . . . . 979

2.5.1.4 Review of Double Beta Decay Experiments, D. $O$. Caldwell, $\left[\mathrm{Cal89}{ }^{* *}\right] \ldots \ldots$. . . . . . . . . 980

2.5.1.5 Concluding Remarks, H.V. Klapdor-Kleingrothaus, $\left[\mathrm{Kla} 91 \mathrm{~b}^{* *}\right] \ldots \ldots . \ldots . . \ldots 981$

2.5.1.6 Double Beta Decay, M. Moe and P. Vogel, [Moe94**] 982

2.5.1.7 Tables of Double Beta Decay Data, V. I. Tretyak an dYu. G. Zdesenko, [Tre95**] . . . . . . . . . . 983

2.5.1.8 Double Beta Decay and Related Topics, H. V. KlapdorKleingrothaus and S. Stoica, [Kla96**] . . . . . . . . 984

2.5.1.9 Double Beta Decay - Physics at Beyond Accelerator Energies, H. V. Klapdor-Kleingrothaus, $[$ Kla96c**] . . . 985

2.5.2 Ionisation and Time Projection Chambers . . . . . . . . . . . 987

2.5.2.1 Direct Evidence for Two-Neutrino Double-Beta Decay in ${ }^{82} \mathrm{Se}$, S. R. Elliott, A. A. Hahn, M. K. Moe, [Ell87a] 989

2.5.2.2 Double Beta Decays and Dark Matters Studied by ELEGANT Detectors, $H$. Ejiri, [Eji97] . . . . . . . . 993

2.5.2.3 Results of a Search for the Two Neutrino Double $\beta$ Decay of ${ }^{136} \mathrm{Xe}$ with Proportional Counters, Ju. $M$. Gavriljuk, V. V. Kuzminov N. Ya. Osetrova and $S$. Ratkevich, [Gav2000**] . . . . . . . . . . . . 998

2.5.2.4 New Limit on Neutrinoless Double $\beta$ Decay in ${ }^{136} \mathrm{Xe}$ with a Time Projection Chamber, H. T. Wong, F. Böhm, P. Fisher, K. Gabathuler, H. E. Henrikson, D. A. Imel, M. Z. Iqbal, V. Jörgens, L. W. Mitchell, B. M. O'Callaghan-Hay, J. Thomas, M. Treichel, J.-C. Vuilleumier and J.-L. Vuilleumier, [Won91] . . . . . . 999

2.5.2.5 Search for Neutrinoless Double- $\beta$ Decay in ${ }^{136} \mathrm{Xe}$ with a Time Projection Chamber, J.-C. Vuilleumier, J. Busto, J. Farine, V. Jörgens, L. W. Mitchell, M. Treichel, J.L. Vuilleumier, H. T. Wong, F. Böhm, P. Fisher, H. E. Henrikson, D. A. Imel, M. Z. Iqbal, B. M. O'CallaghanHay, J. Thomas and K. Gabathuler, [Vui93**] . . . . 1003 
2.5.2.6 Search for $\beta \beta$ Decay in ${ }^{136} \mathrm{Xe}$ Gotthard-Results, $J$. Farine, $[$ Far97**] . . . . . . . . . . . . . . 1004

2.5.2.7 ${ }^{136} X e$ Double Beta Decay from ITEP, $V$. Artemev, $E$. Brakchman, M. Ivanovsky, A. Karelin, V. Kirichenko, V. Knyazev, O. Kozodaeva, V. Lubimov, A. Mitin, V. Nikolaev, T. Tsvetkova and O. Zeldovich, [Art92] . . . 1005

2.5.2.8 Investigation of Double-Beta Decay of ${ }^{100} \mathrm{Mo}$ with the Liquid-Argon Ionization Chamber, V. D. Ashitkov, A. S. Barabash, S. G. Belogurov, G. Carugno, S. I. Konovalov, F. Massera, I. O. Pilugin, G. Puglierin, R. R. Saakyan, V. N. Stekhanov, V. I. Umatov and I. A. Vanshin, $[$ Ash99**] . . . . . . . . . . . . 1010

2.5.2.9 Two-Neutrino Double- $\beta$ Decay Measurement of ${ }^{100} \mathrm{Mo}$, D. Dassié, R. Eshbach, F. Hubert, Ph. Hubert, M. C. Isaac, C. Izac, F. Leccia, P. Mennrath, A. Vareille, C. Longuemare, F. Mauger, F. Danevich, V. Kouts, V. I. Tretyak, Yu. Vassilyev, Yu. Zdesenko, A. S. Barabash, V. N. Kornoukov, Yu. B. Lepikhin, V. I. Umatov, I. A. Vanushin, C. Augier, D. Blum, J. E. Campagne, S. Jullian, D. Lalanne, F. Laplanche, F. Natchez, G. Pichenot, G. Szklarz, R. Arnold, J. L. Guyonnet, T. Lamhamdi, I. Linck, F. Piquemal, F. Scheibling, V. Brudanin, V. Egorov, O. Kochetov, A. Nozdrin, Ts. Vylov, Sh. Zaparov, H. W. Nicholson and C. S. Sutton, $[$ Das95**] . . . . . . . . . . . . . 1011

2.5.2.10 NEMO Collaboration: Latest Results and Perspectives for the Future, A. S. Barabash for the NEMO Collaboration, [Bar97] . . . . . . . . . . . . . 1012

2.5.2.11 New Approach to the Detection of Neutrinoless DoubleBeta Decay, M. K. Moe, [Moe91] . . . . . . . . . . 1019

2.5.3 Scintillation Detectors . . . . . . . . . . . . . . . . . . 1023

2.5.3.1 Large Volume $\mathrm{CdWO}_{4}$ Crystal Scintillators, S. $P h$. Burachas, F. A. Danevich, A. Sh. Georgadze, H. V. Klapdor-Kleingrothaus, V. V. Kobychev, B. N. Kropivyansky, V. N. Kuts, A. Müller, V. V. Muzalevsky, A. S. Nikolaiko, O. A. Ponkratenko, V. D. Ryzhikov, A. S. Sai, I. M. Solsky, V. I. Tretyak and Yu. G. Zdesenko, [Bur96] . . . . . . . . . . . . . . . . 1025

2.5.3.2 Investigation of $\beta^{+} \beta^{+}$and $\beta^{+} /$EC Decay of ${ }^{106} \mathrm{Cd}, F$. A. Danevich, A. Sh. Georgadze, J. Hellmig, M. Hirsch, $H$. V. Klapdor-Kleingrothaus, V. V. Kobychev, B. N. Kropivyansky, V. N. Kuts, A. Müller, A. S. Nikolaiko, 
F. Petry, O. A. Ponkratenko, H. Strecker, V. I. Tretyak, M. Völlinger and Yu. Zdesenko, [Dan96**] . . . . . . 1030

2.5.3.3 New Results of ${ }^{116} \mathrm{Cd}$ Double $\beta$ Decay Study with ${ }^{116} \mathrm{CdWO}_{4}$ Scintillators, F. A. Danevich, A. Sh. Georgadze, V. V. Kobychev, B. N. Kropivyansky, A. S. Nikolaiko, O. A. Ponkratenko, V. I. Tretyak, S. Yu. Zdesenko, Yu. G. Zdesenko, P. G. Bizzeti, T. F. Fazzini and P. R. Maurenzig, [Dan2000**] . . . . . . 1031

2.5.4 Semiconductor Detectors . . . . . . . . . . . . . . . . . 1033

2.5.4.1 Experimental Search for Neutrinoless Double- $\beta$ Decay of ${ }^{100} \mathrm{Mo}, M$. Alston-Garnjost, B. L. Dougherty, R. W. Kenney, R. D. Tripp, J. M. Krivicich, H. W. Nicholson, C. S. Sutton, B. D. Dieterle, S. D. Foltz, C. P. Leavitt, R. A. Reeder, J. D. Baker and A. J. Caffrey, [Als93] . . . . . . . . . . . . . . . 1035

2.5.4.2 Review of Double Beta Decay Results from ${ }^{76} \mathrm{Ge}, D$. O. Caldwell, [Cal86a] . . . . . . . . . . . . 1039

2.5.4.3 Limits on Neutrinoless $\beta \beta$ Decay Including that with Majoron Emission, D. O. Caldwell, R. M. Eisberg, D. M. Grumm, M. S. Witherell, F. S. Goulding and A. R. Smith, [Cal87] . . . . . . . . . . . . . 1054

2.5.4.4 Double Beta Decay - Present and Future, D. O. Caldwell, $\left[\mathrm{Cal91}{ }^{* *}\right] \ldots \ldots \ldots \ldots$. . . . . . . 1058

2.5.4.5 New Possibilities in a Double Beta Decay Experiment Using Enriched ${ }^{76} \mathrm{Ge}$ Inside of an Active $\mathrm{Si}(\mathrm{Li})$ Shielding, L. A. Popeko, A. V. Derbin, I. A. Kondurov, V. V. Martyanov, H. V. Klapdor and J. Metzinger, $[$ Pop86] . . . . . . . . . . . . . . . . . 1059

2.5.4.6 New Results in the ITEP/YePI Double Beta-Decay Experiment with Enriched Germanium Detector, $A$. A. Vasenko, I. V. Kirpichnikov, V. A. Kuznetsov, A. S. Starostin, A. G. Djanyan, V. S. Pogosov, S. P. Shachysisyan and A. G. Tamanyan, [Vas90] . . . . . . 1061

2.5.4.7 Spectroscopy with Enriched Detectors: Double Beta Decay and Perspectives in Astrophysical $\gamma$-Ray Spectroscopy and in Dark Matter Detection, $H$. V. KlapdorKleingrothaus, $\left[\mathrm{Kla90}^{* *}\right]$. . . . . . . . . . . . . 1069

2.5.4.8 The Heidelberg-Moscow Double Beta Decay Experiment with Enriched ${ }^{76} \mathrm{Ge}$ : First Results, H. V. KlapdorKleingrothaus, [Kla92] . . . . . . . . . . . 1070

2.5.4.9 Introductory Remarks - Workshop Session on Double Beta Decay, H. V. Klapdor-Kleingrothaus, [Kla94] . . . 1073 
2.5.4.10 Double Beta Decay - Physics at Beyond Accelerator

Energies, H. V. Klapdor-Kleingrothaus, [Kla96a] . . . 1076

2.5.4.11 HEIDELBERG-MOSCOW $\beta \beta$ Experiment with ${ }^{76} \mathrm{Ge}$ : Full Setup with Five Detectors, HEIDELBERG-MOSCOW Collaboration, M. Günther, J. Hellmig, G. Heusser, M. Hirsch, H. V. Klapdor-Kleingrothaus, B. Maier, $H$. Päs, F. Petry, Y. Ramachers, H. Strecker, M. Völlinger, A. Balysh, S. T. Belyaev, A. Demehin, A. Gurov, I. Kondratenko, D. Kotel'nikov, V. I. Lebedev and A. Müller, [HM97] . . . . . . . . . . . . . . . . 1083

2.5.4.12 Digital Pulse Shape Analysis by Neural Networks for the Heidelberg-Moscow-Double-Beta-Decay-Experiment, B. Majorovits and H. V. Klapdor-Kleingrothaus, $[$ Maj99**] . . . . . . . . . . . . . . 1097

2.5.4.13 Neutrinoless Double- $\beta$ Decay of ${ }^{76}$ Ge: First Results from the International Germanium Experiment (IGEX) with Six Isotopically Enriched Detectors, C. E. Aalseth, F. T. Avignone III, R. L. Brodzinski, J. I. Collar, D. González, E. Garcia, F. Hasenbalg, W. K. Hensley, I. V. Kirpichnikov, A. A. Klimenko, H. S. Miley, A. Morales, J. Morales, A. Ortiz de Solorzano, S. B. Osetrov, V. S. Pogosov J. Puimedon, J. H. Reeves, A. Salinas, M. L. Sarsa, A. A. Smolnikov, A. S. Starostin, A. G. Tamanyan, A. A. Vasenko, S. I. Vasiliev and J. A. Villar, [Aal99a**] . . . . . . . . . . . . 1098

2.5.5.1 Cryogenic Thermal Detectors in Subnuclear Physics and Astrophysics, E. Fiorini, [Fio91a**] . . . . . . 1101

2.5.5.2 Low Temperature Detectors in Nonaccelerator Particle Physics, A. Giuliani, [Giu91**] . . . . . . . . . . 1102

2.5.5.3 The CUORE Project: A Large Observatory for Neutrinoless Double Beta Decay and Other Rare Events, A. Giuliani (CUORE Collaboration), [Giu99a] . . . . 1103

2.5.5.4 Superconducting Granule Detectors, K. Pretzl, $[$ Pre93a**] . . . . . . . . . . . 1110

2.5.5.5 Cryogenic Calorimeters in Astro and Particle Physics, K. Pretzl, $[$ Pre99**] . . . . . . . . . . . 1111

2.5.6 Other Large Source Strength Detectors . . . . . . . . . . . . . . . 1113

2.5.6.1 New Approach to the Search for Neutrinoless Double Beta Decay, R. S. Raghavan, [Rag94] . . . . . . . . 1115

2.5.6.2 KAMLAND Proposal ${ }_{n}$ A. Suzuki (KAMLAND Collaboration), $\left[\mathrm{Suz}^{* *}\right] \ldots \ldots \ldots$. . . . . . . 1119 
2.5.6.3 Solar Neutrinos, Atmospheric Neutrinos and Proton Decays in Super-Kamiokande and KamLAND Project, A. Suzuki (KAMLAND Collaboration), [Suz99a ${ }^{* *}$ ] . . 1120

2.5.6.4 Spectroscopy of Double-Beta and Inverse-Beta Decays from ${ }^{100}$ Mo for Neutrinos, H. Ejiri, J. Engel, R. Hazama, P. Krastev, N. Kudomi, and R. G. H. Robertson, $[$ Eji99b] . . . . . . . . . . . . . . . . 1121

2.6 The Future of Double Beta Decay . . . . . . . . . . . . . . 1125

2.6 .1 GENIUS . . . . . . . . . . . . . . . . . . 1125

2.6.1.1 Double Beta Decay - Physics Beyond the Standard Model Now, and in Future (GENIUS), H. V. KlapdorKleingrothaus, $\left[\mathrm{Kla}^{*}{ }^{*}\right]$ ] . . . . . . . . . 1127

2.6.1.2 A Large Scale Double Beta and Dark Matter Experiment: On the Physics Potential of GENIUS, $H$. $V$. Klapdor-Kleingrothaus and M. Hirsch, [Kla97a] . . . 1128

2.6.1.3 Future Perspectives of Double Beta Decay and Dark Matter Search - GENIUS, H. V. Klapdor-Kleingrothaus, J. Hellmig and M. Hirsch, [Kla98d**] . . . . . 1140

2.6.1.4 Ice Shielding in the Large Scale GENIUS Experiment for Double Beta Decay and Dark Matter Search, H. V. Klapdor-Kleingrothaus and Yu. G. Zdesenko, $\left[\mathrm{Kla98 \textrm {e }}^{* *}\right] \ldots \ldots \ldots \ldots . \ldots \ldots 141$

2.6.2 The Physics Potential of Future Double Beta Decay for Beyond Standard Model Physics . . . . . . . . . . . . . . . . 1143

2.6.2.1 Double Beta and Dark Matter Search - Window to New Physics Beyond the Standard Model of Particle Physics, H. V. Klapdor-Kleingrothaus, [Kla99] . . . . . 1145

2.6.2.2 Neutrino Mass and Magnetic Moment in Supersymmetry without $R$-Parity in the Light of Recent Data, G. Bhattacharyya, H. V. Klapdor-Kleingrothaus and H. Päs, [Bha99**] . . . . . . . . . . . . . . . . 1196

2.6.2.3 Proc. Beyond the Desert: Accelerator and Nonaccelerator Approaches, $H$. V. Klapdor-Kleingrothaus and $H$. Päs, [Kla98f**] . . . . . . . . . . . . . 1197

2.6.2.4 Proc. Lepton and Baryon Number Violation in Particle Physics, Astrophysics and Cosmology, H. V. KlapdorKleingrothaus and I. V. Krivosheina, [Kla99f] . . . . 1198

2.6.2.5 Physics Beyond the Standard Model (WEIN'98), $P$. Herczeg, C. M. Hoffman and H. V. Klapdor-Kleingrothaus, $[$ Her99**] . . . . . . . . . . . 1199 
2.6.2.6 Proc. Beyond the Desert: Accelerator, Nonaccelerator and Space Approaches into the Next Millenium, BEYOND 2000, H. V. Klapdor-Kleingrothaus and I. V. Krivosheina, [Kla2000d $\left.{ }^{* *}\right]$. . . . . . . . 1200

Appendix A Ten Years of Heidelberg-Moscow Experiment

A.1 Die Rätsel um die kleinsten und größten Dimensionen im Kosmos, $H$. V. Klapdor, [Rad86]

A.2 Untersuchung des Doppelbetazerfalls von angereichertem ${ }^{76} \mathrm{Ge}$ zur Bestimmung der Neutrinomasse, Vorschlag eines Experiments, H. V. Klapdor, $\left[\mathrm{Kla}^{*} 7^{*}\right] \ldots \ldots \ldots \ldots \ldots \ldots \ldots \ldots$

A.3 Weak and Electromagnetic Interactions in Nuclei - (Evolution of Nuclear Physics into the Particle Domain), H. V. Klapdor, [Kla87a] . 1234

A.4 Keine Neutrinos beim Doppel-Betazerfall?, H. V. Klapdor, [Rad87] . . . 1237

A.5 Protokoll: Über die Durchführung eines Gemeinsamen Experimentes zur Suche nach dem Doppelbetazerfall von ${ }^{76} \mathrm{Ge}, M P I$, Heidelberg and Kurchatov Institute, Moskau für die Jahre 1988 - 1992, [Pro88] . . . . . 1241

A.6 Letters from the German Ministry of Research and Technology about funding the HEIDELBERG-MOSCOW experiment, September 1988 and October 1988, [Let88]

A.7 Deutsch-Sowjetisches Projekt zur Untersuchung der Neutrinomasse Gestartet, [Bun88] . . . . . . . . . . . . . . . . . . . . 1243

A.8 Deutsch-Sowjetisches Projekt zur Untersuchung der Neutrinomasse Gestartet, H. V. Klapdor, [Kla89] . . . . . . . . . . . . . . . . 1246

A.9 Hauptphase eines Deutsch-Sowjetischen Projektes zur Untersuchung der Neutrinomasse beginnt, [Bun90] . . . . . . . . . . . . . . . . 1247

A.10 Das Letzte Rätsel der Physik, [NEU90] . . . . . . . . . . . . . . . . . . 1249

A.11 Neuer Vorstoß zur Bestimmung der Neutrinomasse, H. V. KlapdorKleingrothaus, $[\mathrm{Kla91}] \ldots \ldots$. . . . . . . . . . . . 1250

A.12 Weitere Eingrenzung der Neutrinomasse über den Doppelten Beta-Zerfall, H. V. Klapdor-Kleingrothaus, [Kla91c] . . . . . . . . . . . 1252

A.13 Unique Crystal 'Grown' in OR Could Play Role in Basic Physics, J. A.

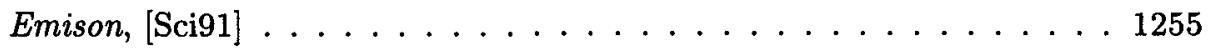

A.14 GRAN SASSO - Enriched Germanium in Action, CERN Courier, [CER91] . . . . . . . . . . . . . . . . . . . 1256

A.15 Suchaktion im Tunnel, H. V. Klapdor-Kleingrothaus, [MPG94] . . . . 1258

A.16 New Experiments Step Up Hunt for Neutrino Mass, D. Normile, [Sci97] 1263

A.17 Status and Perspectives of Double Beta Decay - Window to New Physics Beyond the Standard Model of Particle Physics, H. V. Klapdor-Kleingrothaus, [Kla98a**] and Double Beta Decay with Ge-detectors - and the Future of Double Beta and Dark Matter Search (GENIUS), H. V. Klapdor-Kleingrothaus, $[$ Kla99b**] . . . . . . . . . . . . 1264 
A.18 Ten Years of Heidelberg-Moscow Experiment - a Fresh Look, H. $V$. Klapdor-Kleingrothaus, $[$ Kla2000c] . . . . . . . . . . 1265

Appendix B The Potential Future - GENIUS 1272

B.1 Signals from Beyond the Desert?, CERN Courier, [CER97] . . . . . 1276

B.2 HEIDELBERG, Debut of GENIUS, CERN Courier, [CER97a] . . . . . 1279

B.3 GENIUS - A New Experiment with Large Discovery Potential for Particle and Astrophysics, Proposal, November 20, 1997, first draft, $H$. V. Klapdor-Kleingrothaus J. Hellmig and M. Hirsch, [Kla97c**] . . . 1280

B.4 GENIUS - A Supersensitive Germanium Detector System for Rare Events, Proposal, August 1999, second draft, H. V. Klapdor-Kleingrothaus L. Baudis, G. Heusser, B. Majorovits and H. Päs, [Kla99a**] 1281 